\title{
3D Histological Reconstruction of Fiber Tracts and Direct Comparison with Diffusion Tensor MRI Tractography
}

\author{
Julien Dauguet ${ }^{1}$, Sharon Peled ${ }^{2}$, Vladimir Berezovskii ${ }^{3}$, Thierry Delzescaux ${ }^{4}$, \\ Simon K. Warfield ${ }^{1}$, Richard Born ${ }^{3}$, and Carl-Fredrik Westin ${ }^{5}$ \\ ${ }^{1}$ Computational Radiology Laboratory, Children's Hospital, Brigham and Women's \\ Hospital, Harvard Medical School, Boston, USA \\ ${ }^{2}$ Harvard Center for Neurodegeneration and Repair, Boston, USA \\ ${ }^{3}$ Department of Neurobiology, Harvard Medical School, Boston, USA \\ ${ }^{4}$ Service Hospitalier Frédéric Joliot, CEA, Orsay, France \\ ${ }^{5}$ Laboratory of Mathematics in Imaging, Brigham and Women's Hospital, \\ Harvard Medical School, Boston, USA
}

\begin{abstract}
A classical neural tract tracer, WGA-HRP, was injected at multiple sites within the brain of a macaque monkey. Histological sections of the labeled fiber tracts were reconstructed in 3D, and the fibers were segmented and registered with the anatomical post-mortem MRI from the same animal. Fiber tracing along the same pathways was performed on the DTI data using a classical diffusion tracing technique. The fibers derived from the DTI were compared with those segmented from the histology in order to evaluate the performance of DTI fiber tracing. While there was generally good agreement between the two methods, our results reveal certain limitations of DTI tractography, particularly at regions of fiber tract crossing or bifurcation.
\end{abstract}

\section{Introduction}

Tracing neural connections lies at the heart of neuroanatomy, and has profound implications for the study of neural function and for the study of developmental and adult plasticity of the nervous system. It is also important for the study of neurodegenerative diseases, and for the planning of neurosurgical procedures, such as tumor ablation. Methods for tracing connections in the brain have a long history, beginning with those based on lesions and the resulting retrograde or anterograde degeneration [Nissl 1894; Marchi 1886; Nauta and Gygax 1954]. Subsequent methods exploited the axonal transport of specific molecules, beginning with horseradish peroxidase (HRP) [1, which opened up a new era in neuroanatomy. This was followed by the discovery of a host of other tracers including small fluorescent molecules [23], lectins [4], neurotrophins [5], neurotoxins [6], dextrans [7], and, more recently, carbocyanine dyes [8], latex microspheres [9] and viruses [3]. While constituting a powerful armamentarium with which to study connections in the brain, all of these methods possess the obvious 
drawback that they are highly invasive and require sacrifice of the experimental animal for histological visualization of the transported substance.

In the 1970's, water diffusion magnetic resonance imaging was introduced and later used for medical applications [10]. With the introduction of diffusion tensor imaging (DTI) 11, white matter tractography based on DTI became the first non-invasive, in vivo technique for gross fiber tract tracing. Since then, many advanced methods and models have been proposed to improve the quality of the tractography [121314. It is important to stress the fact that the explicit quantity measured is water diffusion and not fibers. Moreover, since MRI methods in general always obtain a macroscopic measure of a microscopic quantity, which necessarily entails intravoxel averaging, the voxel dimensions influence the measured diffusion tensor at any particular location in the brain.

As the sophistication of DTI tractography algorithms increases, it becomes essential to validate the results. These techniques are already used in many biomedical studies. It is clear that DTI reveals gross connectivity as described in atlases based on histology (e.g. [15]), but, as of yet, no rigorous validation of these techniques has been done. In particular, the performance of DTI tractography is unknown in the case of fiber decussations and the transition of fibers through a relay. Moreover, the ability of the methods to give reliable information on the density of fibers remains an open issue. Here we compare tracts revealed with WGA-HRP on histology to the corresponding tracts calculated from DTI data in a macaque brain. Our group presented preliminary results on this data in [16].

\section{Material and Method}

Using procedures approved by the Harvard Medical Area Standing Committee on Animals, craniotomies were performed on a Macaque monkey under general anesthesia, and 4\% Wheat Germ Agglutinin (WGA) conjugated to HRP (WGA-HRP) in water solution was pressure-injected under microscopic stereotaxic guidance. WGA is a plant lectin that transported in both the retrograde and anterograde directions, and that binds to specific cell-surface glycans, in particular, N-acetylglucosamine and sialic acid, which are then internalized via receptor-mediated endocytosis. Though we use the term in the discussion below, WGA is not a "stain" per se; it is rendered visible by virtue of a reaction with the peroxidase. Four 0.25 microliter injections were made in primary visual cortex (V1), five 0.2 microliter injections each along the pre- and post-central gyri and one 5 microliter injection into the left eye. Three days later the monkey was sacrificed, perfused with aldehyde fixative and the brain removed from the skull.

\subsection{MR Image Acquisition}

Post-mortem MR imaging of the fixed brain was performed on a 4.7T Bruker Biospec using a 3D gradient echo proton density sequence (TR 100, flip angle 10) for anatomy. Diffusion weighted imaging (DWI) was performed using a spinecho sequence (30 directions, bvalues of $1000 \mathrm{~s} / \mathrm{mm}^{2}$ ), with voxel dimensions 
$0.5 \times 0.5 \times 1 \mathrm{~mm}^{3}$. In vivo DWI data was also acquired prior to sacrifice but is not described here.

\subsection{Brain Sectioning and Histo-chemistry}

After MR imaging, the brain was separated into two blocks to facilitate sectioning. The whole brain was serially sectioned in the coronal plane ( $80 \mu \mathrm{m}$ thickness) using a freezing, sliding microtome. Prior to cutting each section, the block face was photographed using a rigidly mounted Hasselblad camera with a 25 Mpixel Phase 1 digital back. This greatly facilitated subsequent 3D reconstruction.

Every eighth section was reacted for WGA-HRP using tetramethyl benzidine [17, producing a series of 90 sections spanning the entire brain. These sections were then mounted on glass slides and scanned using both a GE Healthcare Amersham Typhoon 9410 in fluorescent transparency mode at $100 \mu \mathrm{m}$ resolution, and a regular flat-bed scanner in reflection optical mode at $400 \mathrm{dpi}$ resolution. The fluorescent scanning optimizes the grey/white matter contrast for registration purposes whereas the optical scanning allows differentiation of fiber staining for segmenting the bundles. A linear combination of these two scans produced a hybrid image with both good contrast and clear fiber staining. In this linear combination the optical scanning images were inverted producing white areas of HRP reacted tissue.

\subsection{Photographic Volume and Spatial Alignment}

The block face photographs corresponding to the subset of stained and mounted histological slices were selected and stacked. Within each of the anterior and posterior blocks, the stacked photographs were aligned slice by slice with the flat-bed scanned histology sections. The anterior and posterior blocks were then assembled together using an affine registration. The resulting image data set will be referred to as the "photographic volume."

Each histological slice was then co-aligned on the corresponding photograph using per-hemisphere affine registration, consist of automatically segmenting the hemispheres and estimating an individual 2D affine transformation for each one [18. This volume of histological slices will be referred to as the histological volume. A 3D affine tranformation followed by an elastic Free Form Deformation (FFD) [19] were estimated between the photographic volume and the anatomical MRI data to compensate the mechanical deformations due to the handling of the brain during the two scans [20]. These transformations were applied to the histological volume to get both MRI data and histological volume in the same geometry.

\subsection{Tracing DTI and Histological Volume}

The HRP reacted areas in the histological volume were manually segmented. This was performed by tracing them through the histological slices, and refined by inspecting reformatted orthogonal views. 
The fiber segmentation from the histological volume then was used to as a guide to define seed points for the tractography performed in the DTI volume. A standard hyper-streamline based method for tractography was used [14].

\section{Results}

The three different data sets (the DTI data volume, the photographic volume, and the HRP stained histological volume) were spatially aligned to a common coordinate system to allow for direct comparison between results from DTI tractography and the HRP staining. The histological and photographic volumes were registered to the MRI geometry. Segmentations of fibers according to the staining on the histological slices and the tracts derived from the DTI are presented for comparison for all the injection sites.

In the rest of this section we present five figures, the first four of which show cross-sections of the HRP reacted volume and the segmented fibers overlayed in the structural MRI and photographical volumes. Figure 1 shows sagittal views of the segmented fibers connecting the primary motor cortex (precentral gyrus) and the pons via the internal capsule, along with the fibers connecting the somatosensory cortex (postcentral gyrus) and the ventral posterio-lateral nucleus (VPL) of the thalamus. Figure2 shows fibers in the primary motor cortex (same injection as shown in Figure 1), connecting the left and right hemispheres via the corpus callosum. Figure 3 shows fibers connecting the primary visual cortex (V1) and the lateral geniculate nucleus (LGN) in the thalamus. Figure 4 shows fibers connecting the left eye to the lateral geniculate nucleus (LGN) in the thalamus via the optic chiasm (the optic nerves can not be seen because they are outside of the brain). Figure 5 shows the combined result of the histological tract segmentation and the DTI tractography from Figures 1.4.

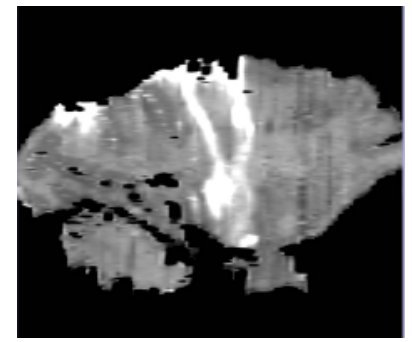

(a)

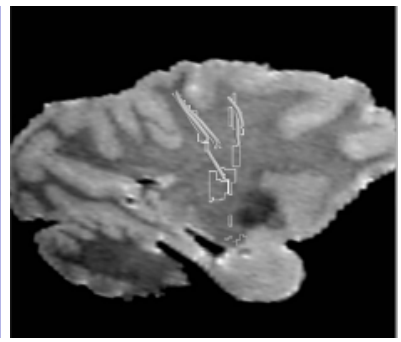

(b)

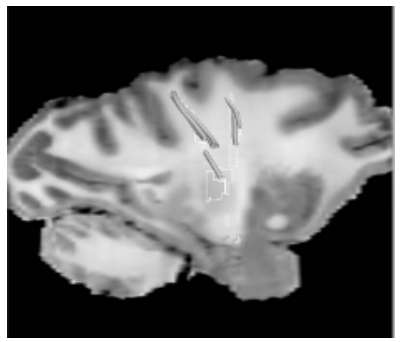

(c)

Fig. 1. Fibers connecting the primary motor cortex (precentral gyrus) to the pons via the internal capsule and fibers connecting the somatosensory cortex (postcentral gyrus) from the ventral posterio-lateral nucleus (VPL) of the thalamus. The figure shows (a) sagittally reformatted view of the HRP reacted histological volume (bright in this picture), (b) fiber pathways derived from histology DTI tractography superimposed on the corresponding structural MRI data and (c) on the photographic volume. 


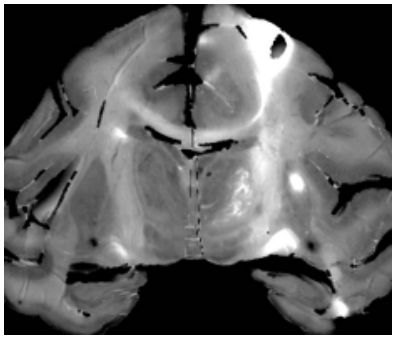

(a)

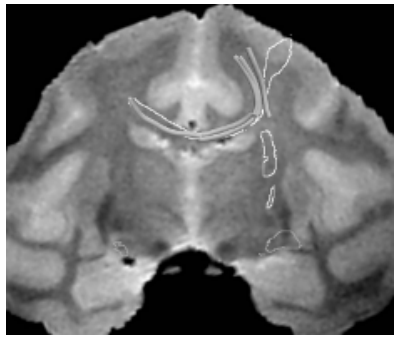

(b)

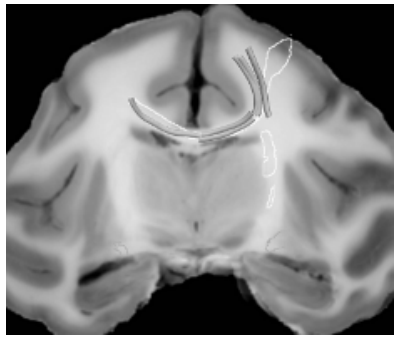

(c)

Fig. 2. Fibers in the primary motor cortex (precentral gyrus) connecting the hemispheres via the corpus callosum. The figure shows (a) coronal view of the HRP reacted histological volume (bright in this picture), (b) fiber pathways derived from histology DTI tractography superimposed on the corresponding structural MRI data and (c) on the photographic volume.

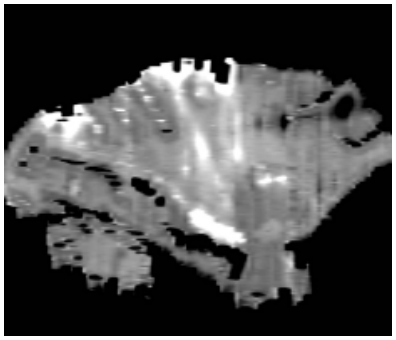

(a)

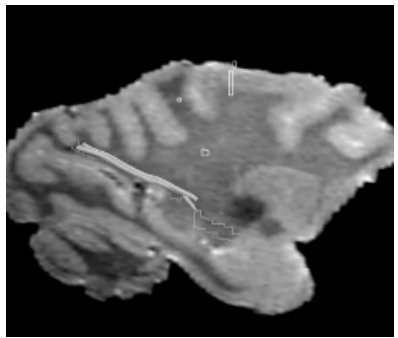

(b)

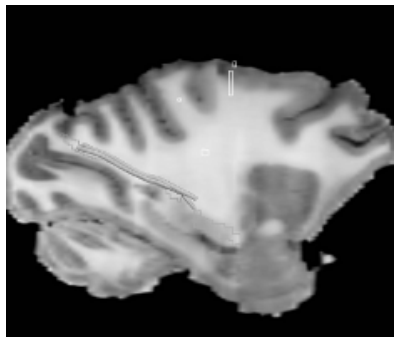

(c)

Fig. 3. Fibers in the primary visual cortex (V1) connecting to the lateral geniculate nucleus (LGN) in the thalamus. The figure shows (a) sagittal view of the HRP reacted histological volume (bright in this picture), (b) fiber pathways derived from histology DTI tractography superimposed on the corresponding structural MRI data and (c) on the photographic volume.

\section{Discussion}

In this paper we have presented results visually comparing tracts derived from DTI tractography and tracts derived from histology sections of a macaque brain. To enable direct comparisons, the data sets were spatially aligned to a common coordinate system using non-linear registration methods. To the best of our knowledge this study is the first that compares long range connectivity derived from histology in 3D and results from DTI tractography.

The results presented show that the tracts derived from DTI correlate well visually with the corresponding tracts derived from the HRP reacted histology volume, as shown in the chiasma (Figure 4) and the connections to the visual cortex (Figure 3). However, some discrepancies can be noticed. For the tract seed points in isotropic areas such as in the cortex, the DTI tractography fails 


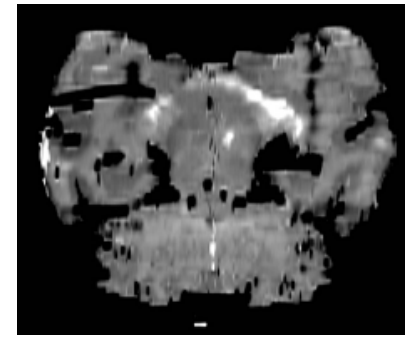

(a)

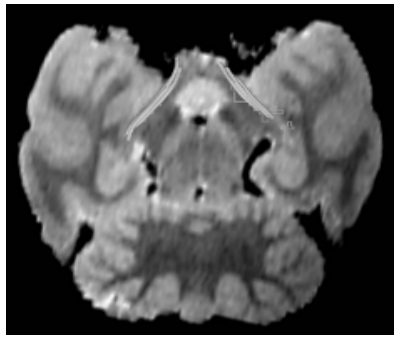

(b)

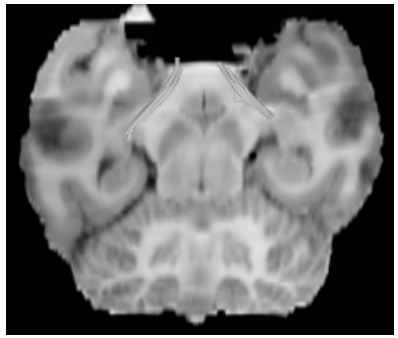

(c)

Fig. 4. Fibers connecting the left eye to the lateral geniculate nucleus (LGN) in the thalamus via the chiasma. The figure shows (a) axial view of the HRP reacted histological volume (bright in this picture), (b) fiber pathways derived from histology DTI tractography superimposed on the corresponding structural MRI data and (c) on the photographic volume.

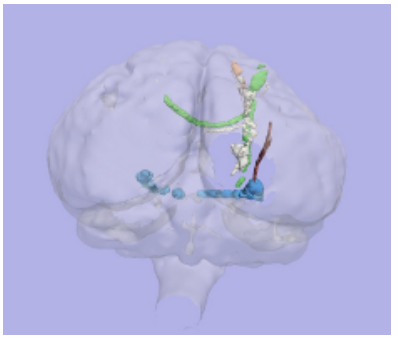

(a)

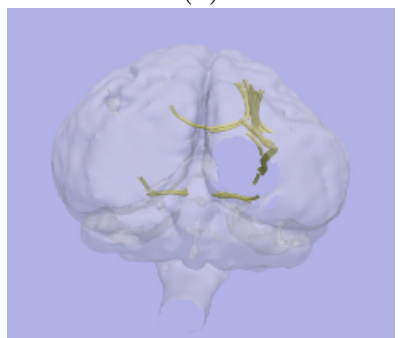

(d)

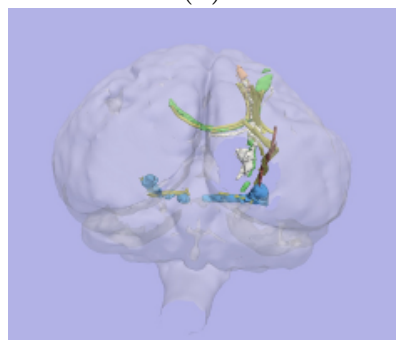

(g)

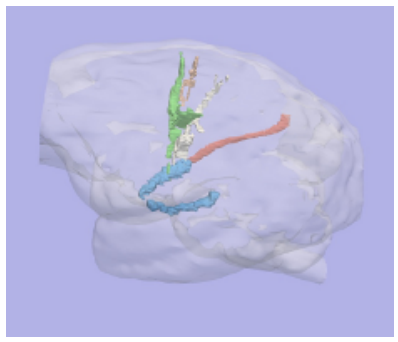

(b)

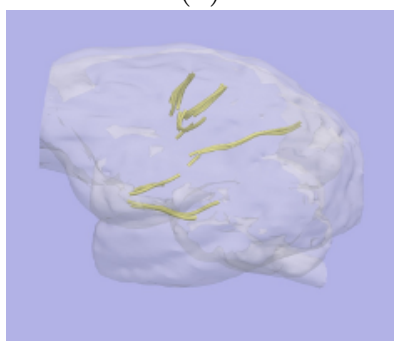

(e)

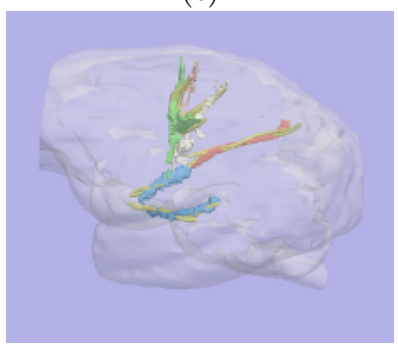

(h)

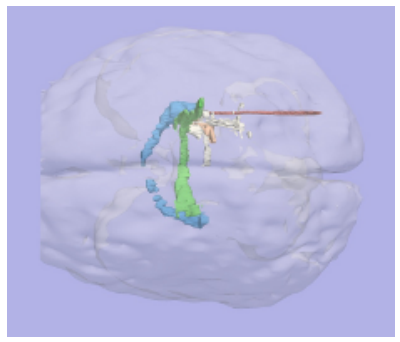

(c)

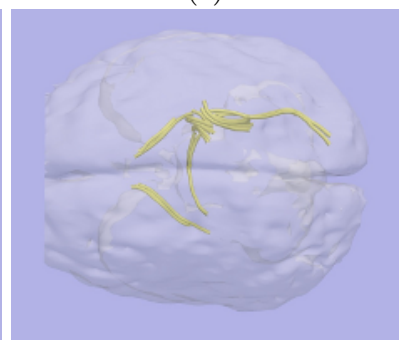

(f)

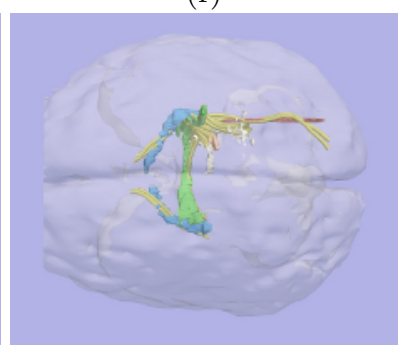

(i)

Fig. 5. Three different views of the paths derived from histological slices (a, b, c), the corresponding fiber tracts derived from DTI (d, e, f), and both results displayed together $(\mathrm{g}, \mathrm{h}, \mathrm{i})$ 
to trace the main bundles. Figure 1) shows that tracts derived from DTI did not continue into the cortex injection sites.

In general, DTI tractography tends to have difficulties when tracts cross or divide. When reaching such areas, the tracts often stop due to low anisotropy of the diffusion tensors or fail to follow the correct branch when tracts divide. This was noticed for the pathways from the primary motor cortex injection going both to the corpus callosum and to the internal capsule. The DTI tractography from the motor cortex injection, shown in Figure 1 and in Figure 2, could not be used to trace both pathways, and additional seed points along the segmented tract were needed. The problem of terminated tracts was noticed when fibers, which were seeded from the optic tract, stopped in the lateral geniculate nucleus (Figures 3 and 4). The resolution of crossing fibers is a known problem in tractography - modeling of two or more tensors in each voxel is an emerging research area that may help in solving this problem [21 22 2324]. Future work will include studies on how to optimize parameter settings for tractography methods, and how to incorporate more complex diffusion models than the single tensor model to better handle complex fiber regions in DTI tractography.

Acknowledgements. The authors gratefully acknowledge Dr. Phillip Hendrickson for his help with the injection and the imaging, and the support of NIH grants P41-RR13218, R01-MH50747, and P30-EY12196. This investigation was also supported in part by NSF ITR 0426558, grant RG 3478A2/2 from the NMSS, and by NIH grants R21 MH67054, P41 RR013218 and U41 RR019703. This work is part of the National Alliance for Medical Image Computing (NAMIC), funded through the NIH Roadmap for Medical Research, Grant U54 EB005149. Information on the National Centers for Biomedical Computing can be obtained from http://nihroadmap.nih.gov/bioinformatics.

\section{References}

1. La Vail, J., La Vail, M.: Retrograde axonal transport in the central nervous system. Science 176 (1972) 1416-1417

2. Bentivoglio, M., Kuypers, H., Catsman-Berrevoets, C., Loewe, H., Dann, O.: Two new fluorescent retrograde neuronal tracers which are transported over long distances. Neurosci Lett. 18(1) (1980) 25-30

3. Kuypers, H., Ugolini, G.: Viruses as transneuronal tracers. Trends Neurosci. 13(2) (1990) $71-75$

4. Schwab, M., Javoy-Agid, F., Agid, Y.: Labeled wheat germ agglutinin (wga) as a new, highly sensitive retrograde tracer in the rat brain hippocampal system. Brain Res. 152 (1978) 145-150

5. Hendry, I., Stockel, K., Thoenen, H., Iversen, L.: The retrograde axonal transport of nerve growth factor. Brain Res. 68 (1974) 103-121

6. Stoeckel, K., Schwab, M., Thoenen, H.: Role of gangliosides in the uptake and retrograde axonal transport of cholera and tetanus toxin as compared to nerve growth factor and wheat germ agglutinin. Brain Res. (1977) 
7. Glover, J., Petursdottir, G., Jansen, J.: Fluorescent dextran-amines used as axonal tracers in the nervous system of the chicken embryo. J Neurosci Methods. 18 (1986) $243-254$

8. Honig, M., Hume, R.: Fluorescent carbocyanine dyes allow living neurons of identified origin to be studied in long-term cultures. J Cell Biol. 103 (1986) 171-187

9. Katz, L., Burkhalter, A., Dreyer, W.: Fluorescent latex microspheres as a retrograde neuronal marker for in vivo and in vitro studies of visual cortex. Nature $\mathbf{3 1 0}$ (1984) 498-500

10. LeBihan, D., Breton, E., Lallemand, D.: MR imaging of intravoxel incoherent motions: application to diffusion and perfusion in neurologic disorders. Radiology 161 (1986) 401-407

11. Basser, P., Mattiello, J., LeBihan, D.: MR diffusion tensor spectroscopy and imaging. Biophys. J. 66(1) (1994) 259-267

12. Mori, S., Crain, B., Chacko, V., van Zijl, P.: Three-dimensional tracking of axonal projections in the brain by magnetic resonance imaging. Ann Neurol. 45 (1999)

13. Basser, P.J., Pajevic, P.J., Pierpaoli, C., Duda, J., Aldroubi, A.: In vivo fiber tractography using dt-mri data. Magn. Reson. Med. (44) (2000) 625-632

14. Westin, C.F., Maier, S., Mamata, H., Nabavi, A., Jolesz, F., Kikinis, R.: Processing and visualization of diffusion tensor MRI. Medical Image Analysis 6 (2002) 93-108

15. Nolte, J.: The Human Brain. An Introduction to Its Functional Anatomy. C.V. Mosby; 5th edition (2002)

16. Peled, S., Berezovskii, V., Hendrickson, P., Born, R., Westin, C.F.: Histological validation of DTI using WGA-HRP in a macaque. In: Proc. ISMRM, Miami (2005)

17. Mesulam, M.: Tetramethyl benzidine for horseradish peroxidase neurohistochemistry: a non-carcinogenic blue reaction product with superior sensitivity for visualizing neural afferents and efferents. J. Histochem Cytochem. 26 (1978)

18. Dauguet, J.: L'imagerie post mortem tridimensionnelle cérébrale. $\mathrm{PhD}$ thesis, École Centrale de Paris (2005)

19. Rueckert, D., Sonoda, L.I., Hayes, C., Hill, D.L., Leach, M.O., Hawkes, D.J.: Nonrigid registration using free-form deformations: Application to breast MR images. IEEE Transactions on Medical Imaging 18(8) (1999) 712-721

20. Delzescaux, T., Dauguet, J., Condé, F., Maroy, R., Frouin, V.: Using 3D non rigid FFD-based method to register post mortem 3D histological data and in vivo MRI of a baboon brain. In: MICCAI 2003. Volume 2879 of LNCS. (2003) 965-966

21. Tuch, D.S.: Diffusion MRI of Complex Tissue Structure. PhD thesis, MIT (2002)

22. Perrin, M., Poupon, C., Rieul, B., Leroux, P., Constantinesco, A., Mangin, J.F., Bihan, D.L.: Validation of q-ball imaging with a diffusion fibre-crossing phantom on a clinical scanner. Phil. Trans. R. Soc. B 360 (2005) 881-891

23. Peled, S., Westin, C.F.: Geometric extraction of two crossing tracts in DWI. In: Proc. ISMRM, Miami (2005)

24. Bergmann, Ø., Kindlmann, G., Lundervold, A., Westin, C.F.: Diffusion k-tensor estimation from q-ball imaging using discretized principal axes. In: MICCAI'06. Lecture Notes in Computer Science, Copenhagen, Denmark (2006) 